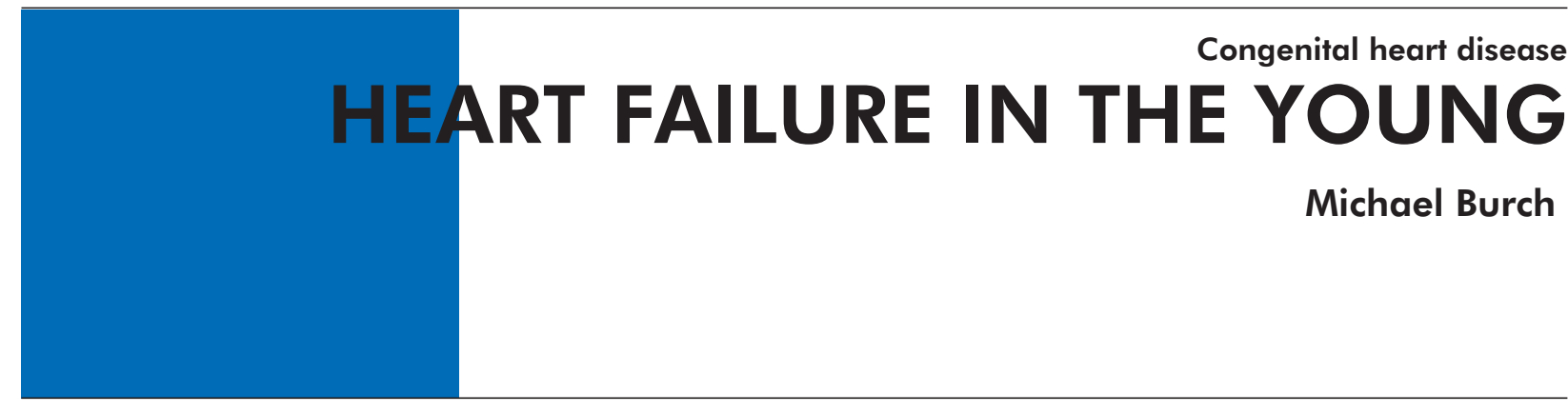

Heart 2002;88:198-202

$\mathrm{H}$ eart failure is an enormous clinical burden in adult medicine, largely because of the prevalence of atheromatous coronary disease. In children, where coronary disease is not the leading cause of heart failure, it is less common. It is, however, an important disease, accounting for $10 \%$ of paediatric cardiac transplants in children.

Cardiac symptoms in children are usually the result of congenital lesions. Most of these lesions, such as septal defects, are amenable to surgical intervention. It is not appropriate to expand on the management of congenital heart lesions in this review. There is a small subgroup of children that have diastolic failure from cardiomyopathic restriction to flow.

The remaining patients, which will be focused on below, have heart failure that is principally related to poor myocardial function and largely comprise those children with dilated poorly contracting ventricles, which can be related to specific aetiologies in some cases. Particular topics of debate in paediatric heart failure concern:

- the diagnosis and management of myocarditis versus dilated cardiomyopathy

- the most appropriate investigations for new onset heart failure

- cellular responses to heart failure

- the increasing population of anthracycline treated survivors of childhood malignant disease

treatment strategies.

\title{
DILATED CARDIOMYOPATHY
}

Indications for transplantation are a guide to the spectrum of causes of severe heart failure. Dilated cardiomyopathy remains the principal indication for cardiac transplantation in children worldwide throughout childhood, apart from infancy when congenital heart disease is a more common indication. The prognosis for dilated cardiomyopathy is around $60 \%$ at five years from presentation (fig 1), with a high attrition within six months of presentation.'

The genetics of dilated cardiomyopathy have been described as a "molecular maze". ${ }^{\text {w1 }}$ Linkage analysis for autosomal dominant dilated cardiomyopathy has proved difficult and direct candidate gene analysis has been used instead, although this is more difficult to use as proof for causation. A variety of lesions have been described including mutations in the cytoskeleton, troponin $\mathrm{T}^{\mathrm{w} 2}$ and other sarcomere protein genes. ${ }^{2}$ For the short term molecular genetic analysis is largely a research tool in dilated cardiomyopathy, but it is likely to enter into clinical practice in the foreseeable future. New onset heart failure in children should be investigated for specific causes and these are discussed below. One particular problem is whether the child has myocarditis or cardiomyopathy as this currently alters management in many centres.

\section{MYOCARDITIS}

Lymphocytic myocarditis accounts for around $10 \%$ of recent onset cardiomyopathy, ${ }^{{ }^{3} 3}$ and this figure may be higher in children. Survival from myocarditis in children and adults is similar at around $80 \%{ }^{3}$ w4

Viruses are the main causes in developed countries, coxsackie B and adenovirus accounting for most cases; Chagas disease is the most common cause in Central and South America, and other infectious causes should be considered. ${ }^{\mathrm{w5}}$ The genetics of the host may determine the outcome. ${ }^{\mathrm{w} 6}$ The majority of infections are insidious, but fulminant infections are well recognised. Perhaps surprisingly, fulminant myocarditis has a better prognosis ${ }^{4}$ as these patients are more likely to develop normal cardiac function if they can be supported through the initial illness. Improved techniques of mechanical support, using biventricular assist devices, has allowed recovery from fulminant myocarditis in children ${ }^{5}$ with complete recovery of cardiac function in three out of four cases. When a biopsy diagnosis of myocarditis is available for children on an assist device it is likely to influence the intensive care team to await recovery, but they may want to bridge to early transplantation if no lymphocytic infiltration is seen and the diagnosis of dilated cardiomyopathy is inferred. Acute or non-fulminant myocarditis is more likely to result in a progressive course with death or transplantation being required. 


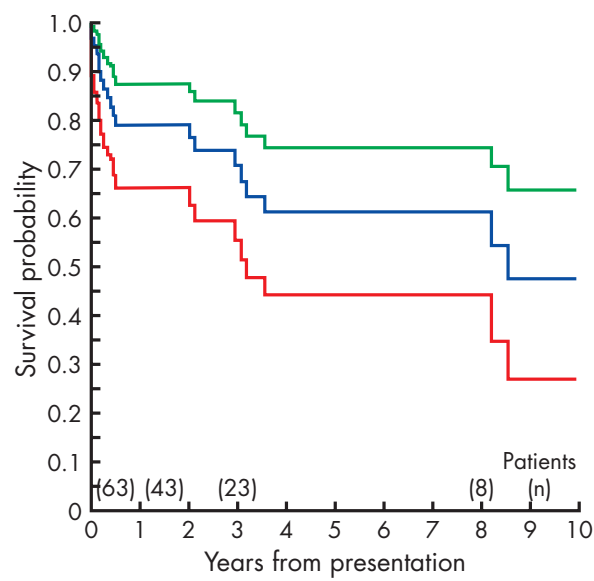

Figure 1 Survival curve (blue line) from presentation of 63 children with dilated cardiomyopathy, with $95 \%$ confidence intervals (red and green lines). Reproduced from Burch et al' with permission from the BM Publishing Group.

The diagnosis of myocarditis is difficult and has historically rested on endomyocardial biopsy evidence of lymphocytic infiltration, yet biopsy in children is not without risk and changes may be patchy. Other tests may be helpful such as assays for autoimmune markers, cardiac troponin $\mathrm{T}$ or $\mathrm{I},{ }^{\mathrm{w} 7}$ and immunocytology may be included in the assessment. Enteroviruses can be looked for using polymerase chain reaction (PCR) from biopsy specimens and from tracheal aspirates. ${ }^{6}$ Paired serology and viral culture are helpful, but are not available early in the course. The ECG is rarely normal, but is not specific. Voltages are reduced and arrhythmias are common, ST changes can be seen, and the findings may mimic myocardial infarction. There are many cases of clinically suspected myocarditis where no supporting evidence is seen.

The treatment of heart failure caused by myocarditis in children is supportive and not essentially different from dilated cardiomyopathy, although patients with fulminant myocarditis are more likely to be supported to recovery rather than transplantation. Much has been written on immune suppression and immune globulin therapy. Studies in children and adults showed promise, ${ }^{7 \text { w8 }}$ but myocarditis has a high rate of spontaneous recovery, and when randomised studies have been performed there has been no advantage to treatment with these strategies. ${ }^{38}$ In paediatrics it is still common practice to treat new onset heart failure with very high dose methylprednisolone and/or immune globulin. Both of these options can result in fluid balance changes that can precipitate worsening failure. Also an improvement in function may not be related to resolution of myocarditis as immune globulins may have a beneficial effect by modulating the effects of cytokines in some cases. ${ }^{9}$ Cytokines cause migration of leucocytes and they are up regulated in heart failure, which may cause inflammation and damage in heart muscle (see below). Therefore an early improvement in systolic function during immunoglobulin therapy should not be assumed to be confirmation of the diagnosis of myocarditis, as it is also seen in dilated cardiomyopathy. There was no long term survival benefit for immunoglobulin therapy in a large controlled study. ${ }^{8}$ Empirically, there seems little reason for immune modulation to work in children if it does not work in young adults with fulminant myocarditis. At present a blanket policy of immune suppression and/or immune globulin in paediatric practice cannot be justified on the evidence base. Until a randomised study is available in children such treatment is not recommended.

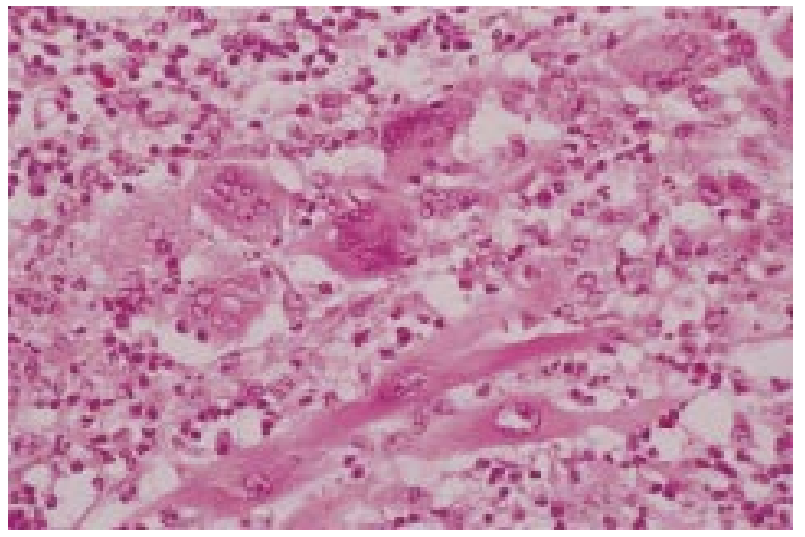

Figure 2 Histological sample from an explanted heart of a 15 year old girl who presented with new onset heart failure and required ECMO (extracorporeal membrane oxygenation) bridge to transplant. Histology shows lymphocytic infiltration with giant cell formationgiant cell myocarditis. The patient remains well at follow up. Slide provided by Dr Marian Malone from the pathology department at Great Ormond Street Hospital for Children.

Immune suppression should not be dismissed in all paediatric myocarditis, as there are specific instances where it is indicated. Giant cell myocarditis ${ }^{10}$ is a rare disease with a characteristic histological appearance (fig 2); these patients appear to benefit from immune suppression. When transplantation is undertaken there is a high risk of recurrence in the transplanted heart, yet it can be effectively treated by increased immune suppression. ${ }^{\mathrm{w} 9}$ Also systemic autoimmune diseases such as systemic lupus erythematosus (SLE) can cause a myocarditis, which will, like the systemic disease, respond to immune suppression. A drug induced/allergic reaction can cause an eosinophilic infiltrate, which may respond to steroids. In addition the role of cellular and humoral immunity in dilated cardiomyopathy has become increasingly implicated in dilated cardiomyopathy. A variety of autoantibodies have been identified, such as those against $\beta$ receptors $^{\mathrm{w10}}$ and cardiac myosin heavy chain. ${ }^{11}$ Recently, immunoglobulin adsorption and IgG substitution has been shown to improve cardiac function clinical status and reduce oxidative stress in dilated cardiomyopathy $\mathrm{y}^{12 \mathrm{w11}}$ and this may be causally related to a reduction in circulating autoantibodies.

\section{INVESTIGATIONS IN NEW ONSET HEART FAILURE IN CHILDREN}

Most paediatric cardiac units have extensive investigation sheets for dilated cardiomyopathy/new onset failure, but there is a degree of scepticism about the chance of turning up a positive result and there is a perception that investigations should not be over extensive. While this is not the view of all involved in the field, it is pragmatic, as it is important that the tests are run thoroughly and results followed up. With too many investigations there may be a tendency for results to be mislaid or overlooked. It is probably wise to target the investigations that are considered most likely.

Cardiomyopathy investigations should vary with the type of heart muscle disease-for example, restrictive, hypertrophic, and dilated. Most new onset heart failure in children is caused by congenital heart disease. Clearly this should be excluded at the initial assessment. It is recognised that an anomalous left coronary from the pulmonary artery will present with a dilated left ventricle and this may cause confusion with a dilated cardiomyopathy. Therefore careful assessment of 
infants with poorly contracting left ventricles should include ECG, echocardiography, and, if there is any doubt, coronary angiography. Metabolic defects should always be considered, as there may be implications for genetic counselling or treatment strategies. Infiltration of the myocardium occurs with inborn errors of metabolism such as glycogen storage diseases, but Fabry's disease, haemochromatosis, and amyloidosis are more common causes of heart failure in adult life, as are damage from toxins such as alcohol, cocaine, and radiation. With infant hypertrophic cardiomyopathy Pompe's disease should be excluded; it can be assessed with initial screening for vacuolated lymphocytes, and confirmed using acid maltase analysis in blood or skin.

Autoimmune diseases are recognised to be associated with cardiomyopathy and these include SLE. It is increasingly recognised that the children of mothers who are anti-Ro positive or anti-La positive may develop cardiomyopathy despite adequate pacing. ${ }^{13}$

Long chain fatty acids supply most of the energy for the heart; they are transported across the plasma membrane and metabolised in the mitochondria. A number of recessive lesions in the proteins required for this have been described. These include carnitine deficiency and medium chain acyl-CoA dehydrogenase deficiency. In general systemic problems such as ammonaemia, acidosis, hypoglycaemia, and coma may be more prominent than cardiomyopathy. Mitochondrial disease typically presents with a hypertrophic and poorly contracting left ventricle. Brain, cardiac, and skeletal muscle function are often affected, as all have a high energy need. Skeletal muscle biopsy shows "ragged red" fibres. Inheritance is through the maternal line. Barth syndrome is an $\mathrm{X}$ linked disorder of mitochondrial function related to a lipid remodelling defect; 3-methylglutaconic aciduria is evident and is often associated with neutropenia, hypercholesterolaemia, hypoglycaemia, and lactic acidosis. The mitochondrial dysfunction of Kearns-Sayre disease syndrome is associated with ophthalmoplegia, retinal pigmentation hearing loss, endocrine dysfunction, and cardiomyopathy with conduction defects. Molecular genetic diagnosis is available for a number of these mitochondrial conditions. Mutations in the gene G4.5 cause a variety of severe infant cardiomyopathies including Barth syndrome and isolated left ventricular noncompaction. ${ }^{\mathrm{w} 12}$

In paediatric practice children with skeletal myopathies such as Duchenne and Becker dystrophy may develop cardiomyopathy, which is initially hypertrophic but becomes dilated. They are suspected clinically before symptomatic cardiomyopathy in the majority of cases. Rarely $\mathrm{X}$ linked deficiency of cardiac dystrophin can be seen without skeletal cardiomyopathy.

Some recommended investigations for new onset heart failure are shown in table 1, including procedures that could reveal mitochondrial disorders and autoimmune disease. In both instances there may be a strong suspicion from other problems arising. Similarly, some rare causes of cardiomyopathy can be suspected from the history of associated disease and extensive tests should only then be undertaken. Thyroid disease, sarcoid, parathyroid disease, phaeochromocytoma, and severe nutritional deficiencies are rare causes of childhood cardiomyopathy and are not recommended for initial screening unless suspected clinically.

\section{APOPTOSIS, CYTOKINES, AND REGENERATION}

The cellular basis of heart failure in children will depend on the cause of ventricular dysfunction, but for most patients apopto-
Table 1 Investigations in new onset paediatric failure (dilated left ventricle)

- Echocardiography (including check for anomalous coronary)

$\checkmark E C G$

- Myocarditis: Tracheal aspirate for viral PCR, paired serology (including coxsackie, adenovirus, echo, influenza, parainfluenza, varicella, RSV, rubella, CMV, EBV, HIV, parvovirus, mycoplasma, and endemic infections depending on geography-for example, Chagas' disease, dengue, diphtheria, Coxiella burnetti; many organisms cause myocarditis, and this list is not exclusive), troponin T, blood count for lymphocytosis. Myocardial biopsy (see text) for histology and PCR. Consider toxins if suggested by history, and illegal drugs (for example, cocaine)

- Autoimmune: Anti-Ro and Anti-La, full SLE screen including antinuclear antibody, double stranded DNA, rheumatoid factor, ESR. Autoantibody screen (availability varies) - for example, anti-mysosin $\beta$ receptor antibodies

Mitochondrial: Carnitine, acyl carnitine, lactate, glucose, white cell count for neutropenia, urine amino acids for methylglutaconic aciduria, muscle biopsy if clinical suspicion of mitochondrial disease. Molecular genetic diagnosis of Barth syndrome is available in some centres

CMV, cytomegalovirus, EBV, Epstein-Barr virus; ESR, erythrocyte sedimentation rate; HIV, human immunodeficiency virus; PCR, polymerase chain reaction; RSV, respiratory syncytial virus; SLE, systemic lupus erythematosus.

sis is likely to be involved. There is strong histopathological evidence that apoptosis is distinct from necrotic cell death, in that it is genetically programmed and is designed to destroy damaged cells that, for example, could become cancerous. There is controversy over whether apoptosis occurs in myocytes, as they are cells which cannot divide, but it is now accepted that it does occur. ${ }^{14}$ Ventricular distension, increased wall stress, and neurohumoral activation upregulate genes such as c-myc, c-fos, and fetal proteins. A fetal metabolic gene profile is seen probably by downregulating adult genes rather than upregulating fetal genes. ${ }^{\mathrm{w} 13}$ Angiotensin II release may stimulate myocyte apoptosis. This leads to release of cytochrome c from mitochondria ${ }^{\mathrm{w} 14}$ and activation of proteolytic capsases, which results in progression of proteolysis. In theory, if the heart is supported the cytoplasmic proteins could recover as the nucleus is initially unaffected. Therefore, left ventricular support is attractive because remodelling, and reversal of neurohumoral abnormalities and wall stress reduction, may lead to cell recovery if the apoptotic process has not become irreversible and damaged the nucleus. Reversal of neurohumoral abnormalities can be achieved with $\beta$ blockers and angiotensin converting enzyme (ACE) inhibitors.

Surgical procedures may aid remodelling. Clearly closure of a ventricular septal defect allows immediate reduction in left ventricular dimensions. In the setting of dilated cardiomyopathy mitral valve surgery has been successfully used to aid remodelling, although early experience in children in our own centre has not been encouraging. The Batista operation is a partial ventriculectomy, ${ }^{15}$ which actively remodels the left ventricle, but it is assumed that the myocytes are functioning well or will recover subsequently. If there is excessive scarring or fibrosis then the outcome of a Batista is less likely to be favourable. There is little experience of the Batista operation in children. The left ventricle can be actively rested by mechanical support, and impeller pumps may allow very long term ambulatory support, although they do involve an incision into the left ventricle.

Cytokines are hormone-like proteins that foster communication between immune cells. Adults with heart failure have 


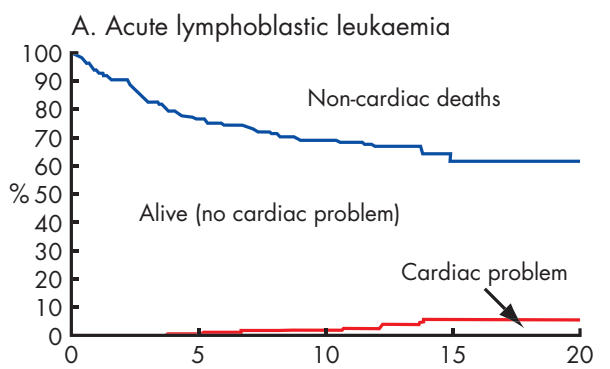

B. Wilms' tumour

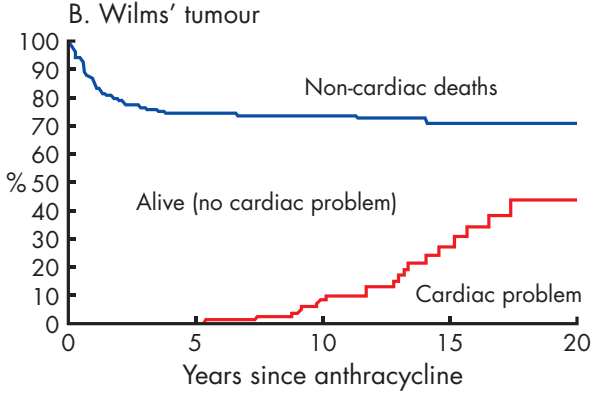

Figure 3 Multistate survival curves for 346 consecutive acute lymphoblastic leukaemia and Wilms' tumour patients treated with anthracycline between 1971 and 1990, identifying non-cardiac deaths and cardiac problems (fractional shortening $<25 \%$ ). Adapted from Sorenson et al. ${ }^{18}$

raised concentrations of cytokines in the circulation, ${ }^{\text {w15 }}$ and tumour necrosis factor $\alpha$ and interleukin 1 and 6 have been examined. The overexpression of these cytokines may play a role in the pathogenesis of heart failure, perhaps by dysregulation of apoptosis, direct effects on calcium dependent processes, and impaired $\beta$ adrenergic signal transduction. Systemic administration of tumour necrosis factor causes myocardial depression. Brain natriuretic peptide is a cardiac peptide that is a useful predictor of survival in heart failure. ${ }^{\text {w16 }}$ Cytokines and vasoactive peptides have not been as extensively investigated in children as in adults. It is possible that immunoglobulin infusion may exert its beneficial effect by modulating cytokines.

Myocyte regeneration has been a controversial issue, but a recent study of chimerism after transplantation does appear to demonstrate that it occurs. ${ }^{16}$ Furthermore, it has been recently demonstrated that human stem cells can differentiate to a cardiomyocyte phenotype in the adult murine heart. ${ }^{\mathrm{w} 17}$ This suggests that the likely source of the chimeric cells posttransplant is the marrow. All of this gives hope for successful regeneration after prolonged support and may signal the beginning of the age of successful stem cell implantation.

\section{ANTHRACYCLINE CARDIOTOXICITY}

About half of all young adults who survive childhood cancer have received anthracyclines, particularly daunorubicin or doxorubicin. The number of young adult patients with potential heart muscle disease is therefore going to be larger than, for example, young adult survivors of tetralogy of Fallot, or transposition. Cardiotoxicity is dose related, and myocyte damage on biopsy has a linear relation with cumulative dose. The myocyte necrosis is irreversible, although echocardiographic improvement in systolic function and recovery from symptomatic heart failure can be seen with treatment in most cases. In children there is progressive, dose related impairment of afterload in asymptomatic individuals. ${ }^{17}$ Age at treatment has an inverse relation with end systolic wall stress. The

\section{Heart failure in the young: key points}

Myocarditis

- Coxsackie and adenovirus account for the majority of cases of viral myocarditis

- Fulminant infections have a better prognosis than nonfulminant infections

- Children can be bridged to recovery using mechanical support

- Randomised studies have not shown any benefit from immune suppression

- Immunoglobulin may reduce cytokines in heart failure and short term echo improvement may be attributable to this, not resolution of myocarditis

Apoptosis, cytokines, and regeneration

- Apoptosis occurs in myocytes

- The nucleus is affected later in myocyte apoptosis and this may allow recovery with bridging

- New myocytes can develop as shown by chimeric studies

- Circulating cytokines such as tumour necrosis factor $\alpha$ are increased in heart failure

Heart failure treatment in children

- ACE inhibitors are widely used in children

- Carvedilol is helpful in stable patients

- Aspirin may oppose the action of ACE inhibitors and carvedilol

longer the interval between treatment and assessment the greater the abnormality of wall stress; as most follow up studies do not extend beyond 20 years, there is a potential for a significant increase in the number of patients with overt disease in middle age.

A prospective longitudinal and actuarial assessment of late daunorubicin and doxorubicin cardiotoxicity in 184 survivors of childhood ${ }^{18}$ cancer has shown that the most important predictor of worsening cardiac performance was total dose with a cut off of $242 \mathrm{mg} / \mathrm{m}^{2}$, above which end systolic wall stress would be expected to deteriorate in a dose related regression. The higher dose used in the Wilms' tumour group was associated with increased cardiac disease at follow up (fig 3). It is reassuring that most paediatric acute lymphoblastic leukaemia patients receive less than this dose currently; however, paediatric solid tumours may receive higher doses.

Epirubicin may be less cardiotoxic. Dexrazoxane is used as a cytoprotective agent in metastatic breast cancer patients who have received $>300 \mathrm{mg} / \mathrm{m}^{2}$ of doxorubicin. It chelates with iron in the myocytes and prevents the formation of the doxorubicin-iron complex that is thought to be cardiotoxic. Probucol, a strong antioxidant, has also been beneficial in animal studies. Activation of poly-ADP ribose polymerase by oxidant mediated DNA damage has been shown to contribute to the cardiotoxicity of doxorubicin. Inhibitors of this nuclear enzyme may offer cardioprotective effects. ${ }^{\text {w18 }}$

\section{PAEDIATRIC TREATMENT FOR HEART FAILURE}

Heart failure treatment in children is very similar to that of adults. However, because of lack of resources and a reluctance of the pharmaceutical industry to undertake trials, few of the proven treatments in adults are licensed in children and paediatric preparations are not available, leaving parents to grind and dissolve adult tablets. The pharmaceutical industry cannot promote products for indications and age groups outside the licence. The implication of this is that product information outside the licence may be difficult to obtain. A principle of paediatric drug usage is that the therapeutic effect in 
children is likely to be similar to what it is in adults, but pharmacokinetics are different. On the whole, paediatric cardiologists follow the evidence base in adults, but the thought that drugs work differently or have a different effect in children leaves the potential for individuals to use idiosyncratic treatments and ignore the evidence base. For example, phosphodiesterase III inhibitors have been shown to have a deleterious effect on long term survival, milrinone having a $28 \%$ increase in mortality compared to placebo. ${ }^{\mathrm{w} 19}$ Yet oral phosphodiesterase inhibitors such as enoximone are still used by paediatric cardiologists.

ACE inhibitors are widely used in children, but $\beta$ blockers are less commonly used even though the adult evidence base is strong; carvedilol has been successfully used in New York Heart Association functional class III and IV patients, ${ }^{19}$ and it has also been used in children. ${ }^{20}$ They should only be introduced in stable patients and the dose increased slowly. $\beta$ Blockers must be stopped if inotropes are needed. Spironolactone has been shown to be beneficial in heart failure (although careful potassium monitoring is necessary if used with ACE inhibitors) yet amiloride is widely used in paediatrics. ${ }^{\text {w20 }}$ Digoxin use remains controversial in adults and children, and although therapeutic benefits have been seen in adults with a reduced hospitalisation rate, overall mortality is not reduced. ${ }^{\mathrm{w} 21}$ This puts it behind $\beta$ blockers and ACE inhibitors, which both prolong survival. Digoxin may not be helpful in acute myocarditis. Anticoagulation with warfarin is difficult in children, but those with severe heart failure are at risk of mural thrombus. There is now evidence that aspirin and other non-steroidal antiinflammatory agents can exacerbate heart failure and may reduce the effect of the diuretics, ACE inhibitors, and $\beta$ blockers. ${ }^{\text {w22 } 23}$ The mechanism probably involves the inhibition of prostaglandin synthesis, increasing peripheral resistance and decreasing renal perfusion. Angiotensin II receptor blockers may have a role in replacing ACE inhibitors when there is an unwanted effect such as a severe cough, but they do not convey a survival benefit in adults ${ }^{\mathrm{w} 24}$ and experience of their use in children has been very limited.

There is a small subgroup of patients with diastolic failure from restriction to inflow, such as restrictive cardiomyopathy. They are probably best managed with $\beta$ blockers and low dose diuretics; anticoagulation may be required as there is a high incidence of embolic disease in this condition.

Ultimately many children with heart failure may deteriorate sufficiently to require mechanical support. For small children extracorporeal membrane oxygenation is used but for older children pneumatic external assists such as the Berlin Heart or the Medos can be used. Both have been used as successful bridges to transplant. The number of paediatric donor organs is closer to the number of children requiring transplantation than is the case in adults, providing bridging is available. This makes long term implantable mechanical support attractive as a bridge to transplant, although it is as yet unclear whether the new impeller pumps can be used in paediatric cardiomyopathy as biventricular pneumatic devices have usually been required. Paediatric transplantation has a five year actuarial survival of approximately $70 \%$ overall since 1982 (for 4419 cases, $^{\text {w25 }}$ with infants having a worse outcome of approximately 60\%). Our unpublished institutional data show that survival is better when adjusted for era and diagnosis, with a worse outcome for complex congenital heart disease, although this latter difference is becoming less obvious with improvements in surgical technique, intensive care, and mechanical support.

\section{CONCLUSIONS}

Paediatric heart failure benefits from the increasing knowledge about the mechanisms of the disease processes. There is potential for significant therapeutic advances in all areas of management in the coming decades.

\section{REFERENCES}

1 Burch M, Siddiqi SA, Celermajer DS, et al. Dilated cardiomyopathy in children: determinants of outcome. Br Heart J 1994;72:246-250.

- This is a review of outcome in dilated cardiomyopathy in children in the UK. The data are useful but are becoming out of date.

2 Kamisago M, Sharma SD, DePalma SR, et al. Mutations in sarcomere protein genes as a cause of dilated cardiomyopathy. $N$ Engl J Med 2000;343: 1688-96.

- An interesting genetic paper that shows evidence for mutations causing dilated cardiomyopathy in different sarcomere proteins.

3 Mason JW, O'Connell JB, Herskowitz A, et al. A clinical trial of immunosuppressive therapy for myocarditis. The myocarditis treatment tria investigators. N Engl J Med 1995;333:269-75.

4 McCarthy RE, Boehmer JP, Hruban RH, et al. Long-term outcome of fulminant myocarditis as compared with acute (nonfulminant) myocarditis. N Engl J Med 2000;342:690-5

5 Stiller B, Dahnert I, Weng YG, et al. Children may survive severe myocarditis with prolonged use of biventricular assist devices. Heart 1999;82:237-40.

6 Akhtar N, Ni J, Stromberg D, et al. Tracheal aspirate as a substrate for polymerase chain reaction detection of viral genome in childhood pneumonia and myocarditis. Circulation 1999;99:2011-18.

7 Drucker NA, Colan SD, Lewis AB, et al. Gamma-globulin treatment of acute myocarditis in the pediatric population. Circulation 1994;89:252-7.

8 McNamara DM, Holubkov R, Starling RC, et al. Controlled trial of intravenous immune globulin in recent-onset dilated cardiomyopathy. Circulation 2001;103:2254-9.

9 Damas JK, Gullestad L, Aass H, et al. Enhanced gene expression of chemokines and their corresponding receptors in mononuclear blood cells in chronic heart failure - modulatory effect of intravenous immunoglobulin. J Am Coll Cardiol 2001;38:187-93.

- An interesting review of the effect of immunoglobulins in heart failure.

10 Cooper LTJ, Berry GJ, Shabetai R. Idiopathic giant-cell myocarditis natural history and treatment. Multicenter giant cell myocarditis study group investigators. N Engl J Med 1997;336:1860-6.

- A helpful report of the outcome in a rare condition.

11 Caforio AL, Grazzini M, Mann JM, et al. Identification of alpha- and beta-cardiac myosin heavy chain isoforms as major autoantigens in dilated cardiomyopathy. Circulation 1992;85:1734-42.

12 Muller J, Wallukat G, Dandel M, et al. Immunoglobulin adsorption in patients with idiopathic dilated cardiomyopathy. Circulation 2000;101:385-91.

13 Nield LE, Silverman ED, Taylor GP, et al. Maternal anti-Ro and anti-La antibody-associated endocardial fibroelastosis. Circulation 2002; 105:843-8.

14 Narula J, Haider N, Virmani R, et al. Apoptosis in myocytes in end-stage heart failure. N Engl J Med 1996;335:1182-9.

- A landmark paper, documenting apoptosis in the heart.

15 Batista RJ, Santos JL, Takeshita N, et al. Partial left ventriculectomy to improve left ventricular function in end-stage heart disease. J Card Surg 1996;11:96-7.

- A paper that launched a new surgical intervention, although a rigorous assessment of the benefits of the technique was awaited.

16 Quaini F, Urbanek K, Beltrami AP, et al. Chimerism of the transplanted heart. N Engl J Med 2002;346:5-15.

- Elegant proof of cardiac regeneration, using sex mismatched transplants.

17 Lipshultz SE, Colan SD, Gelber RD, et al. Late cardiac effects of doxorubicin therapy for acute lymphoblastic leukemia in childhood. N Engl J Med 1991;324:808-15

- An important paper that sparked concern in paediatric oncology units.

18 Sorensen K, Levitt G, Bull C, et al. Late anthracycline cardiotoxicity after childhood cancer: prospective longitudinal and actuarial assessment. Submitted for publication.

- This paper represents a unique long term follow up of a large number of patients with strong statistics.

19 Packer $M$, Coats $A J$, Fowler MB, et al. Effect of carvedilol on survival in severe chronic heart failure. N Engl J Med 2001;344:1651-8.

- A powerful study showing carvedilol can be used safely in late stage heart failure.

20 Bruns LA, Chrisant MK, Lamour JM, et al. Carvedilol as therapy in pediatric heart failure: an initial multicenter experience. J Pediatr $2001 ; 138: 505-11$ 